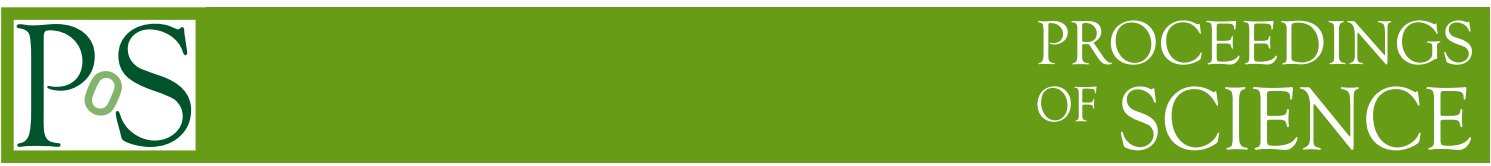

\title{
SUSY search with $b$ jets at ATLAS
}

\section{Marija Milosavljevic, on behalf of the ATLAS Collaboration*}

Institute of Physics, Belgrade

E-mail: marijamephy.bg.ac.yu

\begin{abstract}
Inclusive SUSY searches are typically dominated by squark and gluino production events which are characterised by multiple jets, leptons and missing transverse momentum. Such events may well provide the first evidence of inconsistencies with the Standard Model. In this study signatures which include $b$ jets are analyzed for various SUSY benchmark points using a full GEANT4 simulation of the ATLAS detector both for the signal events and for the Standard Model backgrounds. In the events with $b$ jets isolated leptons may also be present, and all channels with and without leptons are summed. The results presented here are based on a total integrated luminosities of $0.1 \mathrm{fb}^{-1}$ and $1 \mathrm{fb}^{-1}$ that may be collected during the first year of operation of the LHC.
\end{abstract}

2008 Physics at LHC

September 29 - 4 October 2008

Split, Croatia

\footnotetext{
${ }^{*}$ Speaker.
} 


\section{Introduction}

SUSY signals are typically rich in $b$ quarks because the third generation squarks tend to be lighter than the first and the second generation squarks and because Higgsino couplings enhance heavy flavour production. In the benchmark points studied here, the fractions of events containing $b$ jets range from $14.4 \%$ for SU2 to $72.8 \%$ for SU4 point [1]. Given that in QCD events $b$ jets typically occur at the percent level [1], requiring a $b$ jet suppresses the QCD background which may be difficult to control using other techniques.

In this study an analysis of signatures with $b$ jets is performed for SUSY benchmark points SU1, SU2, SU3, SU4 and SU6 using the full detector simulation both for the signal and for the Standard Model backgrounds. Here we include events both with and without isolated leptons. Future studies will consider how best to use the extra information such leptons would provide. Since $b$ jets are usually produced in pairs in SUSY decays, we require at least two such jets in this analysis. More detailed studies of the results found using these Computing System Commissioning (CSC) Monte Carlo samples can be found in [1].

\section{2. $b$ tagging performance in SUSY events}

In this analysis jets were reconstructed using the ATLAS seeded cone algorithm with radius 0.4. Reconstructed jets with $p_{T}>20 \mathrm{GeV}$ and $|\eta|<2.5$ were accepted. The default tagging algorithm based on the 3-dimensional impact parameter and secondary vertex detection [1] was used with weight $>6.75$, giving a nominal $b$ tagging efficiency of $60 \%$. The resulting rejection of light jets (jets not associated with a $b$ or $c$ quark in the Monte Carlo event record) is similar for all of the considered samples and is of order 100. The rejection decreases with increasing $b$ jet $p_{T}$, and so is on average smaller in SUSY events than in $t \bar{t}$ events.

\section{Event selection}

In the full inclusive search with $b$ jets, the 'effective mass' $\left(M_{\text {eff }}\right)$ of the event was defined to be the scalar sum of the missing transverse momentum, the transverse momenta of the four leading jets and all isolated leptons with the $p_{T}>20 \mathrm{GeV}$ and $|\eta|<2.5$. The transverse sphericity $S_{T}$ of the event was calculated from all selected jets and leptons. The cuts used in this analysis are as follows: 1) At least 4 jets in the event with $p_{T}>50 \mathrm{GeV}$; 2) Leading jet $p_{T}>100 \mathrm{GeV}$; 3) Missing transverse energy, $E_{T}^{\text {miss }}>100 \mathrm{GeV}$; 4) Missing transverse energy, $E_{T}^{\text {miss }}>0.2 M_{\text {eff }}$; 5) Transverse sphericity, $S_{T}>0.2$; 6) At least 2 jets are tagged as $b$ jets; 7) $M_{\text {eff }}>600,800$, or $1000 \mathrm{GeV}$. The last cut is used to optimize signal to background ratio in the selected events. It was found that the hardest cut on the effective mass, $M_{\text {eff }}>1000 \mathrm{GeV}$, is the most effective one.

\section{Results}

Events with zero or more leptons and at least two tagged $b$ jets were combined in a single inclusive analysis. After cut 6) the $t \bar{t}$ background dominates as one might expect, but the QCD background remains substantial (Figure 1). The efficiency of the jet plus missing energy trigger 

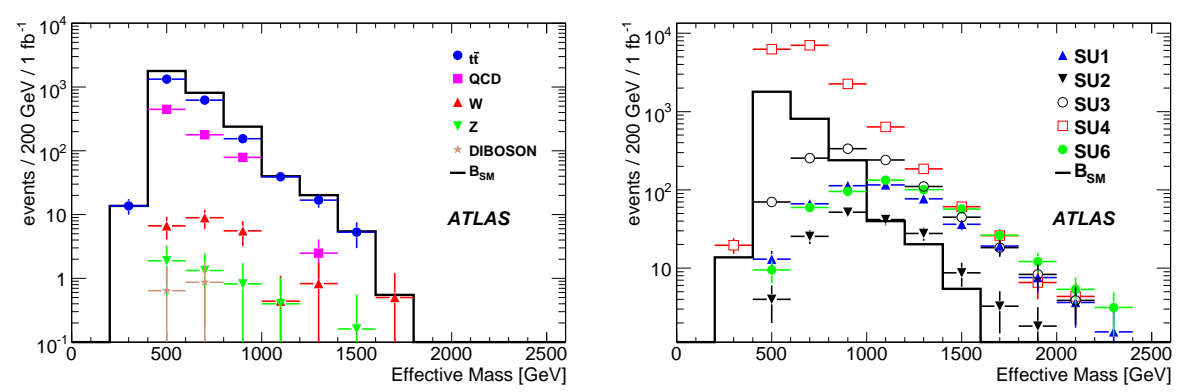

Figure 1: Effective mass distributions for SM backgrounds (left) and SUSY signals with total SM background (right) after cut 6), from [1].

Table 1: $S / B$ ratio and signal significance $Z_{\mathrm{n}}$ including systematic effects for the $b$ jet analysis with $0.1 \mathrm{fb}^{-1}$ and $1 \mathrm{fb}^{-1}$ with $M_{\mathrm{eff}}>1000 \mathrm{GeV}$, from [1].

\begin{tabular}{|c|c|c|c|}
\hline & $S / B$ & $Z_{\mathrm{n}}$ for $0.1 \mathrm{fb}^{-1}$ & $Z_{\mathrm{n}}$ for $1 \mathrm{fb}^{-1}$ \\
\hline SU1 & 3.8 & 6.0 & 9.3 \\
SU2 & 1.3 & 2.3 & 5.0 \\
SU3 & 6.2 & 7.5 & 13.0 \\
SU4 & 13.4 & 12.6 & 21.7 \\
SU6 & 4.9 & 7.1 & 11.2 \\
\hline
\end{tabular}

j70xE70 used in this study is between $97 \%$ and $\sim 100 \%$ for all the considered benchmark points. To calculate the significance in this channel an uncertainty of 50\% for the QCD background and $20 \%$ for the other backgrounds is assumed [1]. The uncertainty on the $b$ tagging efficiency of $60 \%$ is $5 \%$. This is assumed to be included in the $t \bar{t}$ uncertainty and is ignored for the other, smaller backgrounds. Table 1 summarizes the resulting signal significances, $Z_{\mathrm{n}}$, including the above systematic effects [2], for all considered SUSY benchmark points.

\section{Summary}

Events satisfying supersymmetry selection criteria including a pair of $b$ jets and with effective mass higher then $1000 \mathrm{GeV}$ would provide evidence of an excess of SUSY events above the Standard Model backgrounds for the SU3 and SU4 benchmark points with only $0.1 \mathrm{fb}^{-1}$ of data, assuming that the backgrounds can be adequately understood.

\section{References}

[1] ATLAS Collaboration, Expected Performance of the ATLAS Experiment, Detector, Trigger and Physics, CERN-OPEN-2008-020, Geneva, 2008, to appear.

[2] R.D. Cousins and V.L. Highland, Nucl. Instrum. Meth. A320 (1992) 331-335. 